\title{
Piomiositis tropical del músculo ilíaco, obturador interno, piriforme y psoas mayor en un paciente inmunocompetente con claudicación
}

\author{
Tropical pyomyositis of the iliacus, obturator \\ internus, piriformis and psoas major muscles in an \\ immunocompetent patient with claudication
}

\author{
Sánchez-Rodríguez HM,* Morales-Ávalos R, ${ }^{\ddagger}$ Rivera-Zarazúa S, ${ }^{\ddagger}$ \\ Ramírez-Elizondo MT,* Hernández-Rodríguez PA,* Vílchez-Cavazos F, Peña-Martínez VM \\ Hospital Universitario «Dr. José Eleuterio González», Universidad Autónoma de Nuevo León (UANL).
}

\begin{abstract}
RESUMEN. Introducción: La piomiositis es un término que denota infección piógena por lo general primaria de músculo esquelético. Clásicamente descrita de manera frecuente en zonas tropicales y en pacientes inmunocomprometidos; sin embargo, en las últimas décadas se observa cada vez más en zonas de climas templados y con relativa mayor frecuencia en pacientes inmunocompetentes, siendo el grupo etario más afectado el de menores de 30 años. Caso clínico: Masculino de 15 años sin antecedentes médicos de relevancia. Acude por presentar cuadro clínico de siete días de evolución caracterizado por dolor referido en ingle y zona de la cresta ilíaca izquierda asociado a claudicación e hipertermia no cuantificada, que cedían parcialmente a AINES y paracetamol, pero sin lograr mejoría, por lo que es llevado a urgencias por familiares. Conclusiones: Es necesario tener en mente esta patología al momento de realizar el abordaje diagnóstico diferencial de un paciente que se presenta con dolor y limitación funcional de alguna articulación
\end{abstract}

ABSTRACT. Introduction: Pyomyositis is a term that denotes pyogenic infection usually primary skeletal muscle, associated with hematogenous dispersion due to transient bacteremia, or penetrating trauma, usually forming abscesses. Classically described frequently in tropical areas and predominantly affecting the lower limb musculature, however, in recent decades it has increasingly been associated with areas of temperate climates and relatively more frequently in immunosuppressed patients, being the patients under 30 years the most affected. Clinical case: Male of 15 years without relevant medical history. The patient had no history of trauma, falls, surgical interventions, infections or any other systemic condition. He came because of a clinical picture of 7 days of evolution characterized by pain referred to the groin and left iliac crest area associated with claudication and hyperthermia not quantified, which yielded partially to NSAIDs and paracetamol, but without achieving improvement so it is taken emergency by relatives. Conclusions: It is necessary

* Servicio de Infectología, Departamento de Medicina Interna.

¥ Servicio de Ortopedia y Traumatología.

Correspondencia:

Dr. Rodolfo Morales-Ávalos

Av. Francisco I Madero y Dr. Eduardo Aguirre Pequeño S/N, Col. Mitras Centro, C.P. 66460,

Monterrey, Nuevo León, México.

E-mail: rodolfot59@hotmail.com

Recibido: 10-01-2019. Aceptado: 10-02-2021.

Citar como: Sánchez-Rodríguez HM, Morales-Ávalos R, Rivera-Zarazúa S, Ramírez-Elizondo MT, Hernández-Rodríguez PA, Vílchez-Cavazos F, et al. Piomiositis tropical del músculo ilíaco, obturador interno, piriforme y psoas mayor en un paciente inmunocompetente con claudicación. Acta Ortop Mex. 2021; 35(1): 80-84. https://dx.doi.org/10.35366/100936 
asociada a datos sugestivos de un proceso infeccioso o inflamatorio.

Palabras clave: Piomiositis, ilíaco, infección, pediatría, músculo.

\section{Introducción}

La piomiositis es un término que denota infección piógena generalmente primaria de músculo esquelético, asociada a dispersión hematógena debido a bacteriemias transitorias o a traumatismos penetrantes, por lo regular formadora de abscesos. ${ }^{1}$ Clásicamente descrita de manera frecuente en zonas tropicales y con afectación con predominio en la musculatura de los miembros inferiores; sin embargo, en las últimas décadas cada vez más asociado a zonas de climas templados y con relativa mayor frecuencia en pacientes inmunosuprimidos, siendo el grupo etario más afectado el de menores de 30 años así como en hombres sobre mujeres con respecto a la incidencia. Los factores de riesgo más descritos como VIH, diabetes mellitus y trauma, siendo por mucho Staphylococcus aureus el agente más aislado en alrededor de $95 \%$ en los climas tropicales y de $75 \%$ en los templados. ${ }^{2}$

La piomiositis pélvica es una infección piógena aguda o subaguda de los músculos esqueléticos alrededor de la cadera o la pelvis. Los pacientes pediátricos afectados por lo común se presentan con síntomas no específicos como fiebre, irritabilidad, dolor en cadera sin antecedente de trauma, incapacidad para la deambulación y dolor o limitación para la movilidad de la cadera, el diagnóstico con frecuencia se retrasa como resultado de una presentación clínica ambigua y solapante, una variedad amplia de presentaciones clínicas, su baja incidencia y el desconocimiento de la patología por los médicos. ${ }^{3,4}$ Los hallazgos de laboratorio son en general no específicos e incluyen leucocitosis y aumento de los parámetros inflamatorios: velocidad de sedimentación globular (VSG) y proteína C reactiva (PCR). Entre los diagnósticos diferenciales más comunes se encuentran la artritis séptica de cadera, osteomielitis, sinovitis transitoria, formación de abscesos, apendicitis e infecciones gastrointestinales, enfermedad de Perthes, artritis reumatoide juvenil, discitis, trombosis venosa profunda, rabdomiólisis, abscesos perirrenales o del psoas y sarcomas de tejidos blandos. ${ }^{2}$ Presentamos el caso de un paciente de 15 años sin datos sugestivos de inmunosupresión que acudió con un cuadro de una semana de evolución con dolor en cadera izquierda y marcha antálgica.

\section{Reporte de caso}

Masculino de 15 años sin antecedentes médicos de relevancia. No tenía antecedentes de traumatismo, caídas, to bear in mind this pathology when performing the differential diagnostic approach of a patient presenting with pain and functional limitation of some joint associated with data suggestive of an infectious or inflammatory process.

Keywords: Piomyositis, iliac, infection, pediatric, muscle.

intervenciones quirúrgicas, infecciones o algún otro padecimiento sistémico ni viajes recientes fuera de la ciudad, los cuales se indagaron intencionadamente. Presentaba un cuadro clínico de siete días de evolución caracterizado por dolor referido en ingle y zona de la cresta ilíaca izquierda asociado a claudicación e hipertermia no cuantificada, que cedían de manera parcial a AINES y paracetamol, pero sin lograr mejoría. A su llegada el paciente se encontraba hemodinámicamente estable, afebril, con incapacidad para sostener el peso sobre su extremidad inferior izquierda así como rigidez, dolor a la palpación y a la movilidad activa y pasiva de la cadera izquierda con los siguientes rangos de movimiento: flexión de 60 grados, extensión de 20 grados, rotación interna y externa de 15 grados. El paciente no presentaba síntomas en ninguna otra articulación ni cambios de coloración o aumento de temperatura en la piel sobre su cadera, muslo o región glútea. Además, se auscultó un soplo 4/6 en foco mitral y pulmonar sistólico que se irradiaba a cuello, sin encontrar datos de embolización en manos, pies y ojos. También se evidenció un absceso supurativo con base del segundo molar inferior izquierdo, el cual se trató durante su estancia con un lavado, debridación y retiro de pieza dental. El estado inmunológico fue estudiado por el departamento de pediatría realizando pruebas para descartar inmunodeficiencias primarias o secundarias e infecciones virales no encontrando ningún resultado positivo.



Figura 1: Radiografía simple del paciente obtenida en el área de urgencias donde no pueden observarse cambios significativos o alteraciones óseas evidentes. 

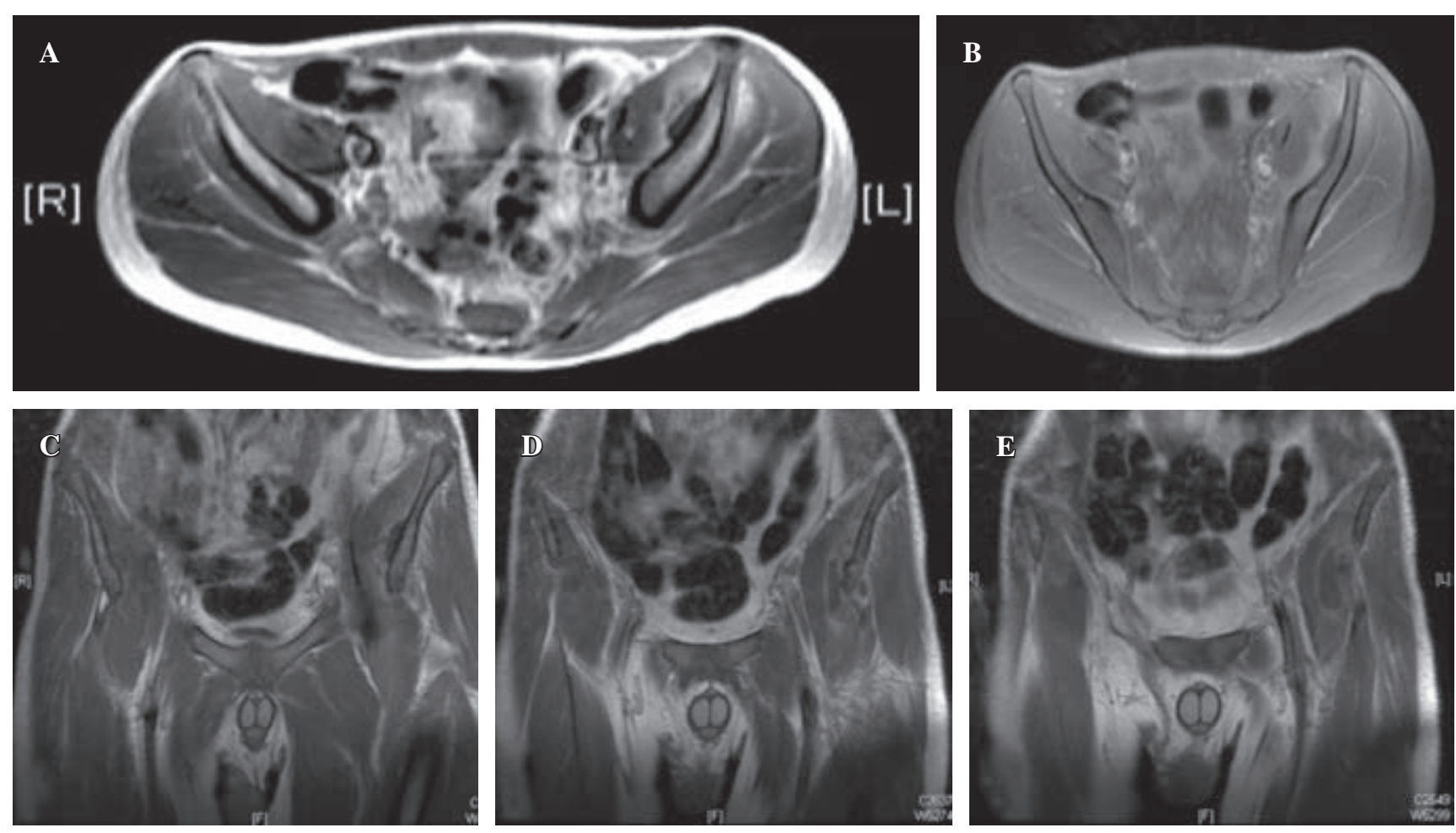

Figura 2: Resonancia magnética nuclear contrastada en la que puede observarse a nivel del músculo ilíaco izquierdo la presencia de una colección en contacto con la tabla interna del hueso ilíaco dentro del espesor y en la superficie del músculo ilíaco izquierdo (A y B). Nótese cómo el absceso se desplaza hacia el vientre del músculo psoas mayor en sentido inferior desde la tabla interna del hueso ilíaco izquierdo (C-E).

En su abordaje inicial se reportaron niveles de leucocitos en $7.54 \mathrm{~K} / \mu \mathrm{l}$, neutrófilos de $5.79 \mathrm{~K} / \mu \mathrm{l}$, velocidad de sedimentación globular en $30 \mathrm{~mm} / \mathrm{hr}$, proteína C reactiva (PCR) de $30.1 \mathrm{mg} / \mathrm{dl}$, las radiografías de pelvis y cadera obtenidas en el área de urgencias no mostraban hallazgos relevantes salvo un borramiento de planos musculares en zona lateral de la cadera izquierda (Figura 1), se realizó una ecografía de articulación de la cadera izquierda, la cual se reportó como normal. Se obtuvieron hemocultivos y se ingresó al paciente para observación y realización de estudios de imagen complementarios con sospecha de un proceso infeccioso o inflamatorio que involucraba la cadera izquierda, el manejo inicial consistió en la administración de líquidos intravenosos, administración de analgésicos opioides por vía intravenosa y reposo en cama.

Se le solicitó una resonancia magnética contrastada de cadera donde se observa una colección adjunta a la tabla interna del hueso ilíaco izquierdo, entre el espesor del músculo ilíaco izquierdo con extensión al músculo piriforme obturador y psoas mayor izquierdo. La lesión se caracterizaba por la presencia de septación y formación de un flemón junto con líquido inflamatorio y edema en el músculo ilíaco izquierdo (Figura 2).

Los resultados de los cultivos sanguíneos (cuatro en total) fueron positivos a las seis horas de su obtención para Staphylococcus aureus sensible a meticilina. Asimismo se le realizó un ecocardiograma transtorácico y transesofágico sin evidencia de vegetaciones; rosa de Bengala, ELISA para VIH, virus de hepatitis C y B también resultaron negativos.
Se inició manejo con antibioticoterapia intravenosa con cefalotina 500 mg IV c/seis horas, logrando mejoría clínica importante a las 24 horas. El paciente presenta una evolución favorable con disminución del dolor, afebril, mejoría en los rangos de movimiento y tolerancia a la carga axial y comienza deambulación asistida a las 48 horas de la administración, la cual realizó por una semana y más tarde movilizado con soporte de peso completo al alta. Respecto a los laboratorios, la PCR disminuyó progresivamente durante su internamiento obteniendo a los 10 días un valor de $3.7 \mathrm{mg} / \mathrm{dl}$, se repitió la toma de nuevos hemocultivos en los cuales se obtuvieron resultados negativos, por lo que se decide su manejo ambulatorio con trimetoprima/sulfametoxazol a dosis de 320/1,600 mg cada 12 horas, paracetamol en caso de dolor y apoyo a tolerancia, con seguimiento en las consultas de ortopedia pediátrica e infectología con nuevos laboratorios y una RMN contrastada de control. En las citas de seguimiento quincenales mostró buena evolución y tras 10 semanas de tratamiento antibiótico persiste asintomático con rangos de movimientos completos y ya reintegrado a actividades deportivas con PCR en $0.5 \mathrm{mg} / \mathrm{dl}$ y una nueva resonancia magnética contrastada en la que se observa una regresión de los cambios inflamatorios en la musculatura pélvica.

\section{Discusión}

La piomiositis es una infección que ocurre predominantemente en áreas tropicales, motivo por el cual también es 
conocida como piomiositis tropical. Nuestro estado es considerado un lugar de clima seco-templado, ${ }^{5}$ en áreas templadas hasta $75 \%$ de los casos de piomiositis ocurren en pacientes inmunocomprometidos, ${ }^{6}$ en comparación con los casos en áreas tropicales donde la mayoría de los casos se dan en pacientes previamente sanos. Entre los principales factores de riesgo predisponentes se encuentra lesión muscular, inmunodeficiencia (ya sea primaria o secundaria), uso de drogas intravenosas, infecciones como VIH, deficiencias nutricionales, diabetes mellitus, enfermedades hematológicas/oncológicas, enfermedades hepáticas, insuficiencia renal, enfermedad pulmonar crónica o enfermedades de tejido conectivo. ${ }^{7,8,9,10,11,12}$ En nuestro paciente no logramos identificar un factor de riesgo claro, a pesar de presentar el antecedente de la fractura, es un antecedente de tres años y en fémur derecho. Sin embargo, el absceso dental podría estar involucrado en el desarrollo de la patología.

El patógeno más común tanto en climas tropicales como templados es Staphylococcus aureus hasta en $90 \%$ de los casos en climas tropicales ${ }^{10}$ y se describe en $60-66 \%$ de los casos en Estados Unidos..$^{12}$ Staphylococcus aureus sensible a meticilina (SAMS) sigue siendo el patógeno más relacionado; sin embargo, ya existen casos reportados de Staphylococcus aureus meticilino resistente (SAMR).$^{12}$ Esta patología tiene tres discretas etapas de presentación. La etapa temprana «invasiva» se caracteriza por dolor difuso e inflamación o edema del músculo afectado con o sin fiebre. La etapa intermedia «supurativa» ocurre de 10 a 21 días después del establecimiento de los síntomas y se caracteriza por la formación de abscesos intramusculares localizados, en esta etapa predominan los síntomas sistémicos como la fiebre y el malestar general así como el dolor y la limitación funcional, del mismo modo ocurre una elevación de los parámetros inflamatorios de laboratorio, $90 \%$ de los pacientes se presentan en esta etapa. La etapa tardía o «septicémica» está asociada a la formación de múltiples abscesos extramusculares, pudiendo cursar con osteomielitis o shock tóxico. ${ }^{10,13,14} \mathrm{El}$ estándar de oro actual para el diagnóstico de esta patología es la realización de una RMN contrastada con gadolinio. Chotai y colaboradores publicaron un reporte de caso de un paciente pediátrico en quien la toma de una primera RMN contrastada no pudo evidenciar la lesión inflamatoria de manera inicial, lo cual contrasta con nuestro reporte en el que la lesión pudo visualizarse sin problemas, siendo este reporte de caso el único en el que la RMN falló en mostrar cambios inflamatorios, hecho relacionado a su toma en etapas muy tempranas de la patología, sugiriendo un período de tiempo de al menos cinco días para el establecimiento de los cambios inflamatorios en la RMN. Sin embargo, la RMN presenta grandes ventajas como una sensibilidad elevada, el no uso de radiación y la posibilidad de localizar abscesos que podrían ser candidatos a drenaje quirúrgico. Las radiografías por lo general no son de mucha ayuda, pero sirven para descartar otras patologías. El ultrasonido y la tomografía contribuyen en la localización de abscesos para así poder realizar drenajes y para descartar otras enfermedades. ${ }^{3}$
En cuanto a la selección de antibioticoterapia, consideramos el trimetoprima/sulfametoxazol debido a que tiene buena biodisponibilidad con buena eficacia contra SAMS e incluso contra cepas de SAMR, pero sensibles a trimetoprima/sulfametoxazol. Al momento no existe evidencia de que las infecciones no necróticas deban ser tratadas con antibióticos que tengan actividad contra la producción de toxinas de Staphylococcus aureus como las Panton-valentine leukocidin (PVL), además tomando en consideración la buena evolución que presentó el paciente..$^{15}$ La duración del tratamiento es por lo regular de tres a cuatro semanas. ${ }^{12,16} \mathrm{Si}$ la patología es reconocida de manera oportuna y tratada agresivamente, la mayoría de los pacientes presentan una recuperación completa sin la aparición de complicaciones a largo plazo. Si se permite que la patología evolucione a estadios avanzados, pueden ocurrir casos de una estancia hospitalaria prolongada, la extensión local al tejido adyacente como el hueso o la articulación (con el subsecuente desarrollo de una osteomielitis o artritis séptica), síndrome compartimental o el desarrollo de una infección remota como pericarditis, endocarditis, miocarditis, formación de abscesos pulmonares o cerebrales, falla renal, septicemia y muerte. ${ }^{14} \mathrm{El}$ índice de mortalidad de esta patología se ha reportado entre 0.9 y $10 \% .^{12}$

\section{Conclusiones}

Es necesario tener en mente esta patología al momento de realizar el abordaje diagnóstico diferencial de un paciente pediátrico con dolor y limitación funcional articular, debido a que el reconocimiento oportuno de la patología y la inmediata instauración de un tratamiento antibiótico son la clave para un desenlace clínico positivo con el rápido restablecimiento de la funcionalidad y así evitar la aparición de complicaciones posteriores.

Referencias

1. Gravot F, Hébert J, Robert-Dehault A, Bouttier R, Le Gall F, Blondin G, et al. Pyomyositis in children: two cases of Staphylococcus aureus infection. Arch Pediatr. 2017; 24 (10): 995-9.

2. Elzohairy MM, Primary pyomyositis in children. Orthopaedics and Traumatology: Surgery and Research. 2018; 104 (3): 397-403. Available in: https://doi.org/1016/j.otsr.2017.12.005.

3. Chotai PN, Hsaiao MS, Pathak I, Banh D, Abdou M, Abdelgawad AA. Pediatric pelvic pyomyositis: Initial MRI can be misleading. J Pediatr Orthop B. 2016; 25 (6): 520-4. doi: 10.1097/bpb.0000000000000295.

4. Kattimani R, McConnell J, Waite J. Pyomyositis of gluteus medius: a case report and review of the literature. J Orthop Case Rep. 2017; 7 (4): 48-50.

5. http://cuentame.inegi.org.mx/monografias/informacion/nl/territorio/ clima.aspx?tema=me\&e=19.

6. Hossain A, Reis ED, Soundararajan K, Kerstein MD, Hollier LH. Nontropical pyomyositis: analysis of eight patients in an urban center. Am Surg. 2000; 66: 1064-6.

7. Lemonick DM. Non-tropical pyomyositis caused by methicillinresistant Staphylococcus aureus : an unusual cause of bilateral leg pain. J Emerg Med. 2012; 42 (3): e55-62.

8. Marimuthu VKN, Midha G, Mukhopadhyay C, Saravu K. Tropical pyomyositis: outcomes and clinical profile. Int $J$ Infect Dis [Internet]. 2016; 45: 142-3. 
9. Small LN, Ross JJ. Tropical and temperate pyomyositis. Infect Dis Clin North Am. 2005; 19: 981-9.

10. Chauhan S, Jain S, Varma S, Chauhan SS. Tropical pyomyositis (myositis tropicans): current perspective. Postgrad Med J. 2004; 80: 267-70.

11. Gibson RK, Rosenthal SJ, Lukert BP. Pyomyositis: increasing recognition in temperate climates. Am J Med. 1994; 77: 768-72.

12. Crum NF, Persons H. Bacterial pyomyositis in the United States. Am J Med. 2004; 117 (6): 420-8.

13. Chiedozi LC. Pyomyositis: review of 205 cases in 112 patients. Am J Surg. 1979; 137 (2): 255-9.

14. Kiran M, Mohamed S, Newton A, George H, Garg N, Bruce C. Pelvic pyomyositis in children: changing trends un ocurrence and management. Int Orthop. 2018; 42 (5): 1143-7.
15. Saeed K, Gould I, Esposito S, Saeed N. Panton-Valentine leukocidinpositive Staphylococcus aureus: a position statement from the International Society of Chemotherapy. Int J Antimicrob Agents. 2018; 51 (1): 16-25.

16. Ticse R, Melgarejo W, Fuentes-Davila A, Ortiz J, Zegarra J. Presentación atípica de piomioisitis tropical difusa de psoas por Staphylococus aureus meticiclino resistente. Rev Peru Med Exp Salud Publica. 2012; 29 (1): 135-38.

Conflicto de intereses: Los autores declaran que no tienen ningún conflicto de intereses.

Financiamiento: Ninguno. 